\title{
Online Life Forecasting Method and State Assessment Architecture of Power Cable Based on Recurrent Neural Network and Internet of Things
}

\author{
Changcheng SONG ${ }^{\mathrm{a}, 1}$, Binglei XUE ${ }^{\mathrm{a}}$, Fumu LU ${ }^{\mathrm{a}}$, Feng LAN ${ }^{\mathrm{a}}$, Zheng YANG ${ }^{\mathrm{b}}$, Chunlei \\ ZHANG $^{\mathrm{c}}$, Peiran YUAN ${ }^{\mathrm{d}}$ \\ ${ }^{a}$ State Grid Shandong Electric Power Company Economic \& Technology Research \\ Institute, Jinan 250021, China \\ ${ }^{b}$ State Grid Jibei Comprehensive Energy Services Co. Ltd, Beijing 100045, China \\ ${ }^{c}$ State Grid Jibei Electric Power Co. Ltd Maintence Branch, Beijing 102487, China \\ ${ }^{d}$ State Grid Jibei Electric Power Co. Ltd Langfang Power Supply Company, Langfang \\ 065000, China
}

\begin{abstract}
For the purpose of increasing the accuracy of power cable life forecasting and status assessment, improving its life cycle management process, this paper proposes a power cable online life forecasting method and status assessment system based on recurrent neural network and Internet of Things (IoTs). Power cable electrical insulation online monitoring system is established on the first place. Then, recurrent neural network and fuzzy analytical hierarchy process are used in the IoTs based power cable online status assessment architecture to proceed life forecasting and status assessment process. Lastly, example analysis is presented to verify the effectiveness and superiority of the methodology introduced in this paper. It is shown that artificial intelligence and IoTs will also have broad development and application prospect when combined with power cable life cycle management.
\end{abstract}

Keywords. power cable, life cycle management, recurrent neural network, Internet of Things (IoTs), life forecasting, status assessment

\section{Introduction}

Power cable is a cable product used to transmit and distribute high-power electrical energy in power systems. Its practical application has a history of more than 100 years. Compared with overhead transmission lines, cables have the advantages of less land occupation, high reliability, large distributed capacitance, less maintenance workload, and low probability of electric shock. Moreover, with the development trend of power systems, cables have the advantages of more favorable conditions for the development of extra high voltage, large capacity and long distance. Therefore, the proportion of cables in the total number of transmission lines is gradually increasing during the development of the power system. XLRE cables have become the first choice for

${ }^{1}$ Corresponding Author: Changcheng Song, State Grid Shandong Electric Power Company Economic \& Technology Research Institute, Jinan 250021, China; E-mail: 18366118336@163.com. 
power cables due to their small quality, large transmission capacity, convenient operation and maintenance, and good electrical and heat resistance properties, since it was researched and developed in 1957 [1].

However, the quality of power cables is affected by the production process, laying methods, operating conditions, and time of use, and the quality is uneven. In addition, the cable production industry itself has serious problems of repeated production, repeated construction, and overcapacity. It will bring great hidden dangers to its safe and reliable operation and bring great inconvenience to residents' lives and economic losses caused by power outages, if the cable is not properly monitored, maintained and managed. Therefore, the full life cycle management of power cables from design and development, production, use and decommission and material recovery can comprehensively consider initial investment and operation and maintenance costs, and improve equipment reliability and efficiency [2]. Life forecasting and condition assessment play a pivotal role in the life cycle management of power cables. It is necessary for electric power companies to conduct real-time condition monitoring of power cables in operation to assess the reliability of their operating conditions, forecast their remaining service life and prevent its possible failures or abnormal conditions, which can achieve the purpose of reducing production costs and improving benefits.

At present, there are few researches on power cable life forecasting and condition assessment around the world. Normal distribution and Weibull distribution is used in reference [3-5] to describe commonly used equipment life and reliability probability distributions. Reference [6] and [7] assessd the operating state of power transformers, and its theories and methods have reference value for the state evaluation of power cables. A differential scanning calorimetry system is used in reference [8] to measure the relationship between the heat flow rate of the sample and the reference and the temperature with the temperature change, thereby obtaining the thermal aging equation, and then forecasting the cable life. Bayesian method used in reference [9] to forecast the insulation reliability of cables. The statistical data of cable insulation aging is used in reference [10] to establish a cable reliability life calculation model that takes into account the failure rate. A status evaluation model is established by setting three levels of cable status in reference [11] based on lognormal distribution, and a corresponding maintenance plan based on the evaluation results is proposed. However, the abovementioned work is mainly based on a specific index. Moreover, for power cables in actual operation of the project, it is impossible to forecast and comprehensively assess their life and status in real time with the above-mentioned methods, or the results of forecasting and evaluation are difficult to reflect the coupling with time. In addition, most of the current methods for cable life forecasting and state evaluation are to assess and calculate the cable in the laboratory after the cable is withdrawn from operation, which is of low practical value. With the continuous increase of data volume and the development of real-time measurement and cloud computing technology, the advantages of artificial intelligence methods and Internet of Things technology have gradually emerged. Artificial intelligence and Internet of Things technology have a good development and application prospects to be applied to the life cycle management of power cables.

An online forecasting method of power cable insulation aging life based on recurrent neural network and a power cable state assessment architecture based on the Internet of Things are proposed in this paper. A power cable online state evaluation architecture based on the Internet of Things is established by building the power cable online state evaluation index system in Section 2. The online forecasting method of 
power cable insulation aging life based on recurrent neural network is put forward in Section 3, including the online forecasting method of power cable insulation aging index based on recurrent neural network and the calculation method of power cable insulation aging life based on fuzzy analytic hierarchy process. The effectiveness of the method is analyzed and proved in Section 4. The conclusion is drawn in Section 5.

\section{Evaluation Index System and Architecture of Power Cable Online Status}

\subsection{Power Cable Online Status Evaluation Index System}

It is generally confirmed that the online index system describing the cable condition assessment mainly determines the cable life by affecting the insulation aging process of the cable. As shown in Figure 1, the online index system mainly includes the following four indices:

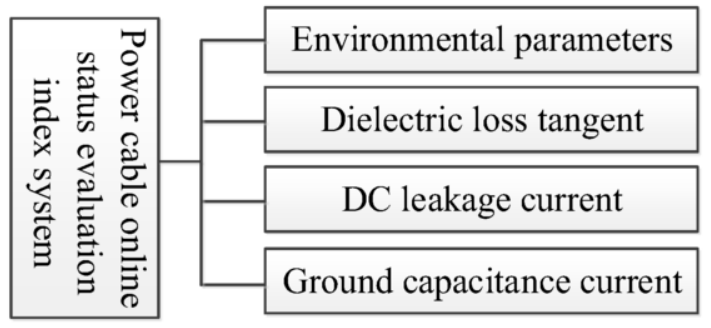

Figure 1. Power cable online status evaluation index system.

1) Environmental parameters $\boldsymbol{E}$ : It mainly includes average surface temperature, average soil moisture, soil $\mathrm{pH}$, road pressure and soil sand quality, which are important factors affecting dielectric insulation.

2) Dielectric loss tangent $\tan \delta$ : It is a characteristic parameter reflecting the dielectric loss of the cable insulation and an important index for evaluating the insulation performance. The $\tan \delta$ will gradually increase with the gradual aging of the cable. The late aging degree will increase significantly, and the $\tan \delta$ will increase significantly until the cable breakdown occurs.

3) DC leakage current $I$ : There will be a nano-ampere DC leakage current in the insulation layer generated by the water tree of the power cable under the action of AC voltage, which is an important parameter for cable insulation testing. Therefore, the water tree aging of the power cable can be found by detecting the DC leakage current of the cable, which reflects the degree of development of the cable insulation aging.

4) Grounding capacitance current $I_{c}$ : This is also an important parameter for cable insulation monitoring. In the aging process of power cable, with the increase of capacitance, the grounding capacitance current of the cable increases.

\subsection{Power Cable Online Status Assessment Architecture Based on the Internet of Things}

In order to assess the operating status of power cables in real time, it is necessary to conduct real-time online monitoring and analysis of the above-mentioned cable operating indices, which is difficult to meet the requirements of online status evaluation because of huge manual measurement workload. To solve this problem, an online state assessment architecture is proposed in this paper, based on the Internet of 
Things technology, as shown in Figure 2. The data collected by the perception layer is uploaded to the data layer through the communication network for data aggregation, integration and analysis, and application services such as management decisions are provided to users based on the results of data analysis and state evaluation.

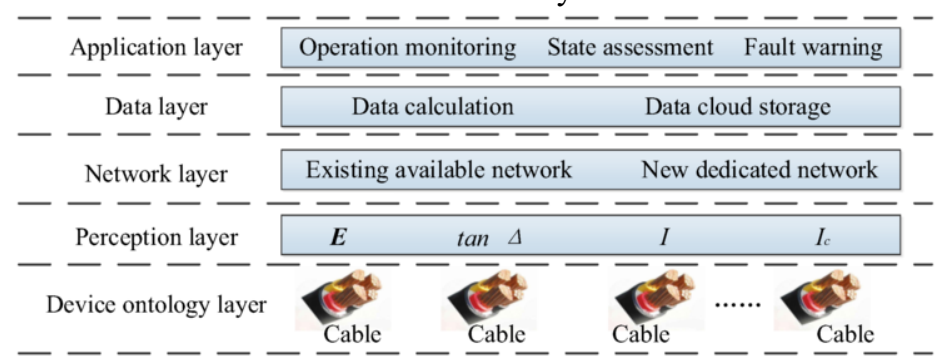

Figure 2. Power cable online state assessment system architecture.

\subsubsection{Perception Layer}

The perception layer is composed of various sensors to collect data based on actual cable equipment operation, including environmental parameters E, dielectric loss tangent $\tan \delta$, DC leakage current I, grounding capacitance current Ic and other data in the index system. The collection terminal supports data collection methods such as periodic, fixed time, current time, etc. The collected data is uploaded to the cloud service platform through the network for data analysis and processing, and the requirements for fast and accurate cable online status evaluation are realized.

\subsubsection{Network Layer}

The network layer uploads the monitoring information data packets collected by the collection terminal through the transmission network to the cloud service platform master station to realize data interaction between on-site collection and the data center. The communication network can directly use the existing network, or build a dedicated network according to the actual situation on site.

\subsubsection{Data Layer}

The data layer is based on the cloud service platform, which integrates and analyzes the data uploaded by each cable device, and obtains the evaluation and analysis results of the operation status of the equipment. Because cloud computing has the characteristics of high flexibility, strong reliability, strong computing power, and strong scalability $[12,13]$, data processing speed and efficiency will be greatly improved; it also depends on the development of cloud storage technology [14], massive monitoring data is stored in the virtual server of the cloud service platform, greatly reducing the cost of data storage.

\subsubsection{Application Layer}

The application layer connects the analyzed and processed data to each application module to realize the user's functional requirements for cable operation monitoring, status evaluation, fault warning, and life forecasting. Thanks to the open architecture of the Internet of Things and the characteristics of integration and decoupling [15], users 
can freely combine and customize background applications according to actual needs, expand their functional applications, and adapt to diverse needs.

\section{Online Forecasting Method of Power Cable Insulation Aging Life Based on Recurrent Neural Network}

\subsection{Online Forecasting Method of Power Cable Insulation Aging Index Based on Recurrent Neural Network}

Neural network is a complex network system that simulates the behavior of the human brain, which is composed of a large number of simple neurons widely connected and layered, as shown in Figure 3. Neural networks are often used to achieve automation, solve complex calculation and forecasting problems, as an important part of artificial intelligence technology [16]. Currently commonly used neural networks mainly include BP neural network, Hopfield network and so on.

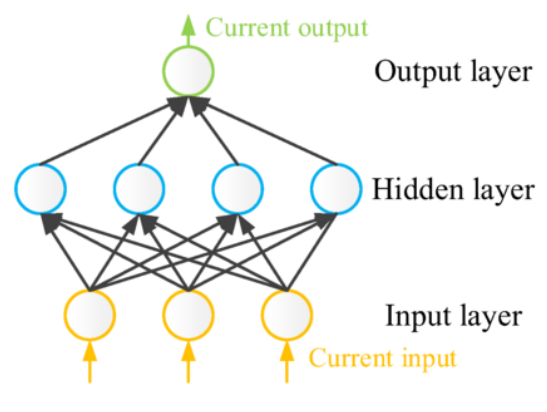

Figure 3. Common neural network structure.

In the above-mentioned more traditional neural network structure, the neurons between the layers are generally fully connected, and the neurons in each layer are not connected. The output vector at the current moment is only related to the input vector at the current moment. However, when this structure is processing time series data (such as real-time data of power cable operating status), the calculation and forecasting results cannot meet the requirement because it cannot reflect the connection and change law of data and time.

Aiming at the shortcomings of common neural networks, a power cable life forecasting method is proposed in this paper, based on recurrent neural networks (RNN). Different from common neural networks, there are connections between neurons in the hidden layers of recurrent neural networks, as shown in Figure 4. The output at the current moment depends not only on the input at the current moment, but also on the system state at the previous moment. In other words, the recurrent neural network will memorize the past system state and use it to calculate the output vector at the current moment, which can better deal with time series problems. 


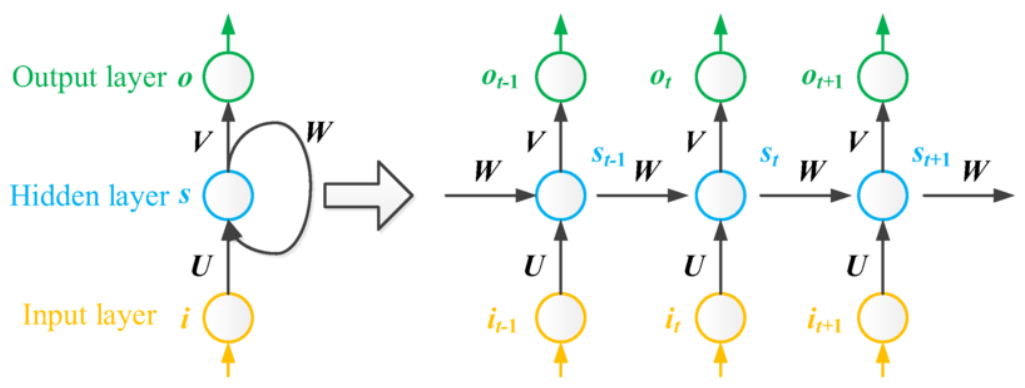

Figure 4. Recurrent neural network structure.

The calculation method of recurrent neural network is:

$$
\boldsymbol{s}_{t}=f\left(\boldsymbol{U} \dot{g}_{t}+\boldsymbol{W} \boldsymbol{g}_{t-1}+\boldsymbol{b}\right)
$$

In the formula, $f$ is the activation function of the neural network, and $\boldsymbol{b}$ is the bias vector. When $t=0, s_{-1}=0$ by default. The calculation method of the output vector $o_{t}$ at time $t$ is

$$
\boldsymbol{o}_{t}=\operatorname{softmax}\left(\boldsymbol{V} \boldsymbol{s}_{t}+\boldsymbol{c}\right)
$$

In the formula, $\boldsymbol{c}$ is the bias vector. The parameter vectors $\boldsymbol{U}, \boldsymbol{V}$, and $\boldsymbol{W}$ of each layer in each time interval are unchanged, which means that the work done by the recurrent neural network at different times is unchanged, which also significantly reduces the number of parameters that need to be learned. The output of the recurrent neural network is related to the input at all previous moments, but the actual calculation takes into account that the connection between the two moments when the interval is too long tends to decrease, so the neural network is generally cut to have a maximum length. In other words, the output at a certain moment is related to all the inputs in a specific period of time before.

For a given power cable, $I$ and $I_{c}$ can be calculated by installing a current sensor on the cable grounding wire, measuring the cable grounding wire current signal, and performing analog-to-digital conversion and discrete Fourier transform. The dielectric loss tangent $\tan \delta$ can be obtained by setting a voltage sensor to obtain the operating voltage of the power cable. After filtering and amplification, analog-to-digital conversion and fast Fourier transform, the fundamental wave of the two signals can be obtained, and the phase difference of the two fundamental waves can be further calculated to obtain the dielectric loss tangent $\tan \delta$. Environmental parameters can be directly measured by installing temperature, humidity, pressure and other sensors around the cable. However, considering that environmental factors also affect other indices to determine the remaining aging life of the cable, they are not considered in the application of the method.

For indices such as $\tan \delta, I$, and $I_{c}$, the real-time measurement and calculation results are used to form time series data, and the time interval between two adjacent moments is $\Delta t$. According to the currently measured data, the recurrent neural network is used to forecast the future index change data. The cut-off length of the neural network is set to $l$, which means that the index data at a certain moment is strongly 
correlated with the index data at the previous $l$ moments. For example, the learning process of $\tan \delta$ of the recurrent neural network is shown in the following formula:

$$
\begin{aligned}
& \boldsymbol{s}_{t}=f\left(\boldsymbol{U} \boldsymbol{g}_{\tan \delta, t}+\boldsymbol{W} \boldsymbol{g} t_{t-1}+\boldsymbol{b}\right) \\
& \boldsymbol{o}_{\tan \delta, t}=\operatorname{softmax}\left(\boldsymbol{V} \boldsymbol{s}_{t}+\boldsymbol{c}\right)
\end{aligned}
$$

In the formula, $\boldsymbol{i}_{\text {tan } \delta, t}=\left[\tan \delta_{t-l}, \tan \delta_{t-l+1}, \ldots, \tan \delta_{t-1}\right]^{\mathrm{T}}$ is the input vector of the dielectric loss tangent at time $t, \boldsymbol{o}_{\tan \delta, t}=\tan \delta_{t}$ is time $t$ The output vector of the dielectric loss tangent.

\subsection{Calculation Method of Power Cable Insulation Aging Life Based on Fuzzy Analytic Hierarchy Process}

Fuzzy analytic hierarchy process is often used to solve the problem of weight distribution among multiple evaluation indices [17], and its steps are as follows:

1) The final decision-making goal and each influencing factor are layered according to the relationship to obtain a hierarchical structure diagram.

2) Construct the fuzzy judgment matrix $\boldsymbol{A}=\left(a_{i j}\right)_{\mathrm{n} \times \mathrm{n}}$, the element $a_{i j}$ in the matrix represents the importance of index $i$ relative to index $j$, which can be obtained by evaluating the index importance according to the $0.1 \sim 0.9$ scale method [17], and satisfy $a_{i j}+a_{j i}=1(i, j=1,2, \ldots, n)$, and the scaling rules are shown in Table 1.

\begin{tabular}{|c|c|}
\hline Scale result & Scale value \\
\hline Both indices are equally important. & $a_{i j}=a_{j i}=0.5$ \\
\hline$i(j)$ is slightly more important than $j(i)$. & $a_{i j}\left(a_{i j}\right)=0.6 \quad a_{j i}\left(a_{i j}\right)=0.4$ \\
\hline$i(j)$ is more important than $j(i)$ & $a_{i j}\left(a_{j i}\right)=0.7 \quad a_{j i}\left(a_{i j}\right)=0.3$ \\
\hline$i(j)$ is much more important than $j(i)$. & $a_{i j}\left(a_{i j}\right)=0.8 \quad a_{j i}\left(a_{i j}\right)=0.2$ \\
\hline$i(j)$ is extremely important than $j(i)$. & $a_{i j}\left(a_{i j}\right)=0.9 \quad a_{j i}\left(a_{i j}\right)=0.1$ \\
\hline
\end{tabular}

Table 1. $0.1 \sim 0.9$ scaling rule.

3) In order to meet the requirements of consistency of thinking, the fuzzy judgment matrix is transformed by the following formula to obtain the consistency matrix $\boldsymbol{B}$ :

$$
\begin{gathered}
b_{i}=\sum_{m=1}^{n} a_{i m}, \quad i \in\{1,2, \cdots, n\} \\
b_{i j}=\left(b_{i}-b_{j}\right) /[2(n-1)]+0.5
\end{gathered}
$$

4) The weight ki of the evaluation index $\mathrm{i}$ is calculated according to the following formula:

$$
\begin{aligned}
& k_{i}=\frac{1}{n}-\frac{1}{n-1}+\frac{2}{n(n-1)} \sum_{m=1}^{n} b_{i m} \\
& i \in\{1,2, \cdots, n\}
\end{aligned}
$$


In this research, the measured index data in section 2.1 should be normalized at first to obtain the relative aging degree of the cable represented by various indexes, as shown in the following formula:

$$
u_{i}=\frac{\left|U_{i}-U_{i f}\right|}{\left|U_{i o}-U_{i f}\right|}
$$

In the formula, $U_{i}$ represents the measured value of index $i, U_{i f}$ represents the qualified threshold of index $i, U_{i o}$ represents the initial value of index $i$, and $u_{i}$ is the normalized index value that characterizes the relative aging of the cable. The larger the value of $u_{i}$, the lighter the aging of the power cable. Finally, the calculation method of the power cable life index is shown in the following formula:

$$
y=\sum_{i=1}^{n} k_{i} u_{i}, \quad i \in\{1,2, \cdots, n\}
$$

In the formula, the larger the value of $y$, the longer the remaining life of the cable. The calculation method of the remaining life of the power cable is shown in the following formula:

$$
T_{S}=\frac{y}{1-y} T_{R}
$$

In the formula, $T_{S}$ represents the remaining life of the cable, while $T_{R}$ represents the operating time of the cable.

The remaining aging insulation life of the cable can be obtained online according to the method shown in equations $(5) \sim(10)$ after the various index data are forecasted. The overall architecture of the method is shown in Figure 5. After obtaining the forecasting results of various indices, the root mean square error (RMSE) between the forecasted value of the index and the true value should be calculated according to formula (11) to assess the accuracy of the forecasting. 


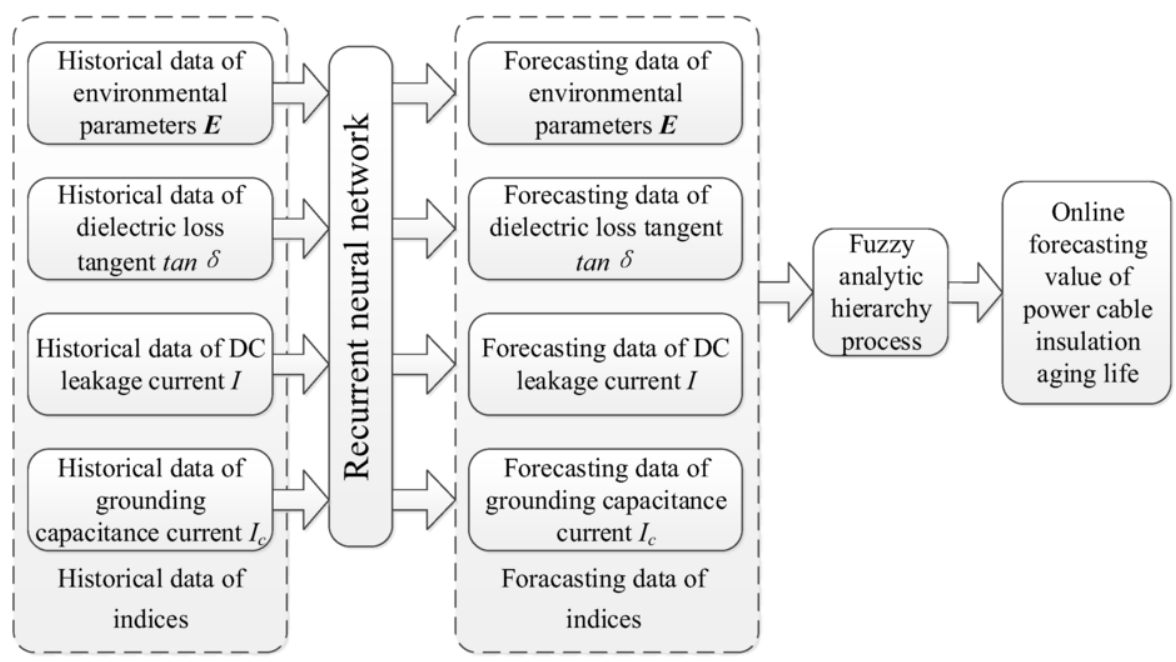

Figure 5. Overall structure of the forecasting method.

$$
\operatorname{RMSE}(\boldsymbol{X})=\sqrt{\frac{1}{N} \sum_{t=1}^{N}[\boldsymbol{X}(t)-\hat{\boldsymbol{X}}(t)]^{2}}
$$

In the formula, $\boldsymbol{X}$ is the set of forecasted values at each time of a certain index, $N$ is the number of forecasted time, $\boldsymbol{X}(t)$ is the forecasted value of index $\boldsymbol{X}$ at the $t$-th time, and is the true value of the index $\boldsymbol{X}$ at the $t$-th time.

\section{Case study}

A 110kV voltage grade XLPE power cable put into operation on Sept. 16, 2007 is used as the test cable in this paper. The neural network toolbox in MATLAB R2014a is used to build a recurrent neural network (RNN) architecture on a computer with a CPU model of Intel(R) G4600@3.60GHz and a RAM model of 8GB. The real-time monitoring data of the power cable between January 1, 2010 and December 31, 2017 is selected for neural network training and learning. The sampling interval is 1 hour, and a total of 70128 sets of data are used.

The cut-off length of the recurrent neural network is set to 48, which means that the index value at a certain moment is related to the index value in the previous 48 hours. The index data between January 1, 2018 and December 31, 2019 is forecasted and the forecasting results are compared with the common BP neural network (BPNN) forecasting results. The online forecasting results of various indices are shown in Figure 6. In order to show the changes of the indices more clearly, the figure only lists the index values at the beginning of each month from January 1, 2018 to December 31, 2019. The comparison of the RMSE between the forecasted value and actual value of each index is shown in Table 2. 

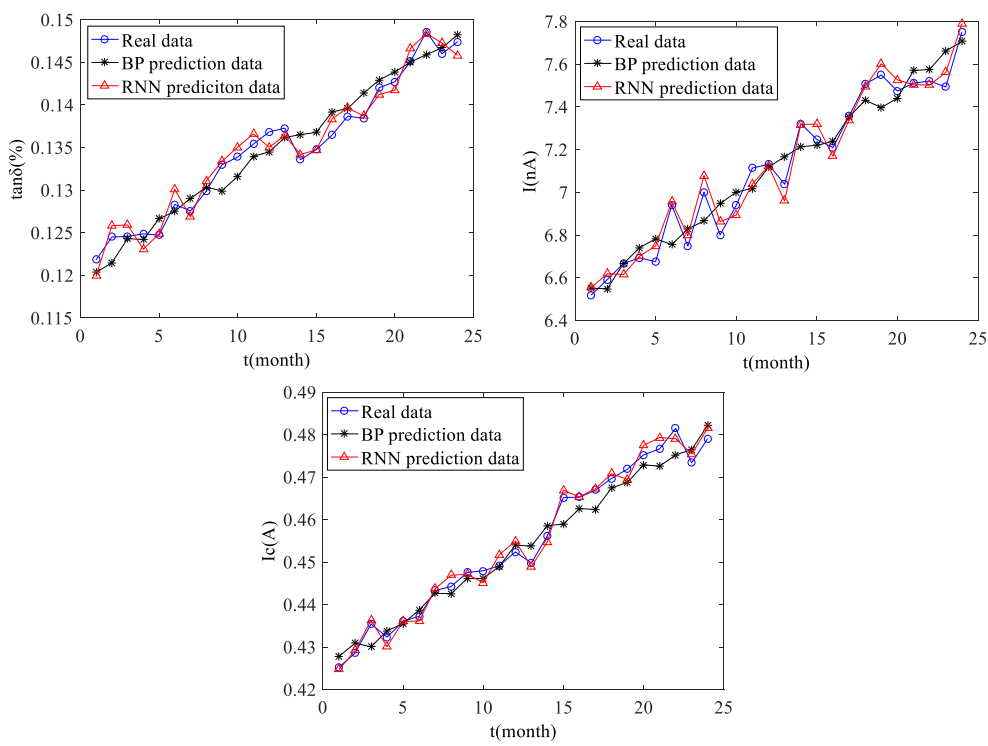
(a) Forecasting results of $\tan \delta$
(b)Forecasting results of $I$ results of $I_{c}$
(c)Forecasting

Figure 6. Online forecasting results of insulation aging indices for power cables.

Table 2. Comparison of RMSE of index forecasting results.

\begin{tabular}{cccc}
\hline & $\tan \delta$ & $I$ & $I_{c}$ \\
\hline RNN & 0.0012 & 0.048 & 0.0018 \\
BPNN & 0.0018 & 0.093 & 0.0032 \\
\hline
\end{tabular}

As Shown in Figure 6 and Table 2, the forecasting method based on RNN proposed in this paper can accurately forecast various insulation aging indices of power cables online, and the accuracy is higher than that of the common BPNN. The effectiveness and superiority of this method are proved.

The method proposed in section 3.2 is used to calculate the remaining life of the power cable. The dielectric loss tangent $\tan \delta$ is the most important index reflecting the degree of power cable aging; the measurement of DC leakage current $I$ is subject to external interference and is less important; the importance of grounding capacitance current $I_{c}$ is between the above two indices. The fuzzy judgment matrix is constructed:

$$
\boldsymbol{A}=\left[\begin{array}{lll}
0.5 & 0.8 & 0.7 \\
0.2 & 0.5 & 0.7 \\
0.3 & 0.3 & 0.5
\end{array}\right]
$$

According to formulas (5) and (6), The consistency matrix is calculated as:

$$
\boldsymbol{B}=\left[\begin{array}{ccc}
0.5 & 0.65 & 0.725 \\
0.35 & 0.5 & 0.575 \\
0.275 & 0.425 & 0.5
\end{array}\right]
$$

According to formula (3), the weight matrix of each index is calculated as:

$$
\boldsymbol{K}=\left[\begin{array}{lll}
0.4583 & 0.3083 & 0.2333
\end{array}\right]^{T}
$$


Finally, the forecasting results of various indices combined, according to formulas (7) (10). The remaining insulation aging life of the power cable is about 11 years and 4 months by the end of 2019 .

\section{Conclusion}

An online forecasting method of power cable insulation aging life based on recurrent neural network and a power cable status assessment architecture based on the Internet of Things are proposed in this paper, aiming at the disadvantages of poor comprehensiveness and low practicability of the existing research on power cable life forecasting and condition assessment. The power cable online state evaluation index system is constructed. Combined with the power cable online state assessment architecture based on the Internet of Things, the method of recurrent neural network is used to forecast the various insulation aging indices of power cables online, and to assess the operating state of the power cable. According to the forecasted results, the fuzzy analytic hierarchy method is used to calculate the remaining insulation aging life of the cable, so as to systematically manage the life cycle of the power cable. Simulation results proves the effectiveness and superiority of the method proposed in this paper. This method and assessment framework apply artificial intelligence and Internet of Things technology to the life forecasting and state assessment of power cables, which have good development and application prospects for the life cycle management of power cables.

\section{References}

[1] A Madariaga, J Martı'n, I Zamora, S Ceballos, O Anaya-Lara. Effective assessment of electric power losses in three-core XLPE cables [J]. IEEE Transactions on Power Systems, 2013, 28(4): 4488-4495.

[2] S Raghavan, B Chowdhury. State diagram-based life cycle management plans for power plant components [J]. IEEE Transactions on Smart Grid, 2015, 6(2): 965-972.

[3] A H Etemadi, M Fotuhi-Firuzabad. Design and routine test optimization of modern protection systems with reliability and economic constraints [J]. IEEE Transactions on Power Delivery, 2012, 27(1): 271278.

[4] C Zhou, M Michcl, D Hcpburn, et al. Online partial discharge monitoring in MV underground cables [J]. IFT Measurement and Science, 2009, 3(5): 353-363.

[5] H Qiao, P Chris. Estimation of the three parameter Weibull probability distribution [J]. Mathematics and Computers in Simulation, 1995, 39(1-2):173-185.

[6] S Li, S Liu, Y Huang. Application of the levenber-marquardt method to determine the rainfall parameters [J], Arch \&Tech, 2004, 40(1): 85-90.

[7] E Pang, G Tang, $\mathrm{H} \mathrm{Yu.} \mathrm{Comparative} \mathrm{research} \mathrm{on} \mathrm{transformer} \mathrm{trust} \mathrm{and} \mathrm{safety} \mathrm{index} \mathrm{[J].} \mathrm{Power} \mathrm{System}$ Protection and Control, 2014, 2(23): 139-145.

[8] J Crine. Cable life predictions from breakdown and accelerated aging tests: influence of frequency and high fields [C]. 2009 IEEE Electrical Insulation Conference, May 31, Montreal, Canada 2009.

[9] Chiodo E, Fabiani D. Bayes inference for reliability of $\mathrm{HV}$ insulation systems in the presence of switching voltage surges using a weibull stress-strength model [C]. IEEE Bologna PowerTech Conference, June 23-26, Bologna, Italy, 2003.

[10] Stotzel M, Zdrallek M. Reliability calculation of MV-distribution networks with regard to ageing in XLPE-insulated cables [J]. IEE Proceeding-Generation Transmission and Distribution, 2001, 148 (6): 597-602.

[11] Q Hong, D Wei, W Yuan, H Yuan, H Guo, Z Wang. Research on cable condition evaluation method based on lognormal distribution [J]. Power System Protection and Control, 2018, 46(2): 79-84 (in Chinese).

[12] P Porambage, J Okwuibe, M Liyanage, M Ylianttila, T Taleb. Survey on multi-access edge computing for internet of things realization [J]. IEEE Communications Surveys \& Tutorials, 2018, 20(4): 2961 2991. 
[13] Z Zhang, X Zhang. Realization of open cloud computing federation based on mobile agent [C]. 2009 IEEE International Conference on Intelligent Computing and Intelligent Systems, Nov. 20-22, Shanghai, China, 2009.

[14] Q He, Z Li, X Zhang. Analysis of the key technology on cloud storage [C]. 2010 International Conference on Future Information Technology and Management Engineering, Oct. 9-10, Changzhou, China, 2010.

[15] H Dang, N Krommenacker, P Charpentier, D Kim. Toward the internet of things for physical internet: Perspectives and challenges [J]. IEEE Internet of Things Journal, 2020, 7(6): 4711 - 4736.

[16] Z Shi. Artificial intelligence [M]. Beijing: China Machine Press, 2016 (in chinese).

[17] Z Wang. A Representable uninorm-based intuitionistic fuzzy analytic hierarchy process [J]. IEEE Transactions on Fuzzy Systems, 2020, 28(10): 2555-2569. 